\title{
The Promising Role of TGF- $\beta /$ SMAD4 in Pancreatic Cancer: The future targeted therapy
}

\author{
Suniida Ahmed', Christopher Schwartz' ${ }^{1}$ M. Zahidunnabi Dewan', Ruliang Xu'*
}

'Department of Pathology, NYU Langone Medical Center, New York, NY 10016, USA

\section{Article Info}

\section{Article Notes}

Received: June 18, 2018

Accepted: May 29, 2019

\section{${ }^{*}$ Correspondence:}

Dr. Ruliang Xu, MD, Ph.D., Department of Pathology, NYU Langone Medical Center, 550 First Avenue, New York, NY 10016, USA; E-mail: xur01@yahoo.com.

(c) 2019 Xu R. This article is distributed under the terms of the Creative Commons Attribution 4.0 International License.

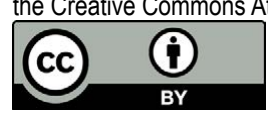

\section{Keywords:}

TGF- $\beta$

Smad4

Pancreatic Cancer

\begin{abstract}
The transforming growth factor $\beta$ (TGF- $\beta$ ) superfamily includes a group of structurally related cytokines that regulate a wide variety of biological processes. The diversity of its action depends on the dynamic tissue microenvironment, and interplays among the factors involving in the signaling pathway. Although its expression is tightly regulated in normal tissue, overexpression of TGF- $\beta$ has been identified in multiple tumor types, including pancreatic adenocarcinoma. In pancreatic tumorigenesis, TGF- $\beta$ acts initially as a tumor suppressor through phosphorylation and activation of SMAD4/DPC4 gene. Mutation of SMAD4/ DPC4 gene results in carcinogenesis and tumor progression in many cancers like lung, colon, and pancreatic cancer. SMAD4 mutation is now recognized in $>50 \%$ of pancreatic ductal carcinomas. This review is to evaluate the diagnostic and prognostic value targeting the TGF- $\beta /$ SMAD4 pathway in pancreatic ductal adenocarcinoma.
\end{abstract}

\section{Introduction}

Pancreatic ductal adenocarcinoma (PDAC) is the fourth leading cause of cancer-related death in the United States, with a median survival rate of fewer than six months and five-year survival rate ranging from $20 \%-30 \% \%^{1-3}$. Several etiologic factors have been shown to be associated with PDAC including advanced age, smoking, longstanding chronic pancreatitis, obesity, and diabetes mellitus. Despite advances in both surgical and neoadjuvant treatment, there remains no definitive prognostic marker/molecular target to improve overall survival. The aggressive nature of PDAC is thought to be a result of multiple key genetic aberrations in genes such as KRAS, p16, TP53, and SMAD4. Among these mutations, inactivation of SMAD4 has been found in $55 \%$ of pancreatic adenocarcinoma cases and thus is similarly known as DPC4 (deleted in pancreatic cancer) ${ }^{4}$. SMAD4 is a member of a large protein family found on chromosome 18q that serves as the main signal transducer for the TGF- $\beta$ family. The majority of SMAD4 mutations are due to either homozygous deletion of both alleles or intragenic mutation in one allele with subsequent "second hit" of the second allele. ${ }^{5-7}$ Thus, the purpose of this review is to elaborate on the role of SMAD4 in pancreatic carcinogenesis and explore its role as both a prognostic and therapeutic marker.

\section{Pancreatic Intraepithelial Neoplasia to Adenocarcinoma}

Pancreatic ductal adenocarcinoma follows a malignant progression through a known precursor lesion in most cases, akin to colorectal carcinogenesis. The known precursor lesion is pancreatic intraepithelial neoplasia (PanIN), which proceeds through histological and molecular changes associated with cytological and 
architectural atypia to progress to adenocarcinoma. Kirsten RAt sarcoma viral oncogene homolog (KRAS) mutation is an early driver event that leads to constitutive activation of multiple pathways, whereas inactivation of SMAD4 is a late event in carcinogenesis ${ }^{8}$. In the early stage of pancreatic carcinogenesis, down-regulation of SMAD4 results in loss of TGF- $\beta$ /SMAD4-dependent cell cycle arrest and apoptosis ${ }^{9}$. Although the role of SMAD4 in tumor progression and metastasis is complex and still under investigation, recent studies on genetically engineered mice with combined SMAD4/KRAS activation mutation developed an aggressive form of PDAC with poor prognosis ${ }^{10}$.

To highlight the importance of multiple genetic mutations driving PDAC oncogenesis, conditional deletion of SMAD4 was insufficient to induce either PanIN or invasive cancer ${ }^{11-13}$. It has been found that SMAD4 loss markedly promotes tumor development initiated by Kras G12D activation and Kras G12D/SMAD4-/tumors exhibit both increased proliferation and tumor stromal formation ${ }^{11,14}$. Furthermore, SMAD4-/- tumors metastasized more frequently than SMAD4 wildtype tumors ${ }^{15}$. Additional studies on in-vivo pancreatic cancer cell lines also found SMAD4 mutation or inactivation is always seen in combination with other gene alterations in K-ras, P53, and/or P16 ${ }^{16}$.

\section{TGF- $\beta$ Signaling}

In normal physiologic conditions, TGF- $\beta$ proteins are involved in the regulation of critical cellular functions including tissue differentiation, cell proliferation, migration, apoptosis, immune surveillance, and maintaining tissue homeostasis ${ }^{17}$. However, TGF- $\beta$ is often overexpressed and activated in inflammation, fibrosis and even tumorigenesis. The dual function of tumor suppression and oncogenesis in the tumor microenvironment is through activation of cell signaling pathways. Active TGF- $\beta$ induces a linear signaling pathway through activation of type II and type I receptor kinase. The ultimate response resulting from SMAD dependent and SMAD independent responses and ligandinduced transcription ${ }^{18}$.

\section{SMAD-Dependent pathway}

The TGF- $\beta$ superfamily consists of TGF- $\beta$ s, bone morphogenetic proteins (BMPs), activins and related proteins ${ }^{18}$. These proteins regulate their effects through the induction of signaling pathways. Among these proteins, TGF- $\beta$ regulates linear signaling pathway either by SMADmediated response or SMAD-independent response. Stored form extracellular and inactive TGF- $\beta$ is a complex form of TGF- $\beta$ pro-peptide and latent transforming growth factor $\beta$ binding protein (LTBP) ${ }^{19}$. Activation of LTBP releases active TGF- $\beta$ s and allow it to bind with receptors (TGF- $\beta$ Rs) to initiate the signaling response. The most important downstream intracellular signaling pathway is
SMAD dependent. TGF- $\beta$ Rs are type I (TGF- $\beta$ RI), type II (TGF- $\beta$ RII), and type III (TGF- $\beta$ RIII). TGF- $\beta$ RI and TGF- $\beta$ RII are transmembrane serine-threonine kinase receptors, consist of extracellular ligand domain, a transmembrane domain, and an intracellular serine-threonine kinase domain. Released mature TGF- $\beta$, following binding with the ligand domain form a TGF- $\beta$ RI/TGF- $\beta$ RII heteromeric complex, subsequently autophosphorylate TGF- $\beta$ RII and transphosphorylate TGF- $\beta \mathrm{RI}^{20}$. Phosphorylation of TGF$\beta R I$ leads to activation of its kinase domain, significant step to phosphorylate SMAD proteins; specifically, R-SMAD (receptor-regulated SMAD) in SMAD dependent pathway ${ }^{20,7}$. Once phosphorylated, R-SMAD associates with the common SMAD/SMAD4, and mediate nuclear translocation of the heterotetrameric complex ${ }^{21}$. The SMAD complex accumulates in the nucleus and directly regulates the expression of different target genes, such as integrin, E-cadherin, collagen, and others ${ }^{22,23}$. It is thought that this TGF- $\beta /$ SMAD4 pathway serves as a tumor suppressor in the early stages of PDAC by promoting cell cycle arrest and apoptosis of epithelial cells ${ }^{18}$. However, persistent overexpression of TGF- $\beta$ changes the tumor microenvironment to switch them from a tumor suppressor to an oncogene ${ }^{24,25}$. This change could be a result of the loss of SMAD4 (30\% PDAC with SMAD4 homozygous deletion) or due to inactivation $(20 \% \text { of PDAC })^{26}$. Downregulation of SMAD4 in the TGF- $\beta$ signaling pathway switch over the action of cell cycle arrest and apoptosis in epithelial cells ${ }^{27}$.

Like TGF- $\beta$, BMPs are complex proteins play an important role in early and late embryonic development and maintain tissue homeostasis. Upon activation, BMP induces, interaction with surface receptors; type I and type II, mediates activation of serine/threonine kinase activity and formation of heteromeric receptor complex ${ }^{28}$. The transduced extracellular signal induces phosphorylation and activation of intracellular signaling of specific proteins including the receptor regulated SMAD proteins (SMAD1, SMAD5, and SMAD8). Activated R-SMAD form complex with Co-SMAD and regulate gene transcriptional response. BMP receptor activation also induces SMAD-independent signaling pathway by activation of intracellular p38 and JNK MAP kinases and small GTPases ${ }^{29}$.

\section{SMAD-Independent pathway}

In SMAD independent or non-canonical pathway TGF$\beta R I$ complexes induce signaling through activation of other pathways such as tumor necrosis factor (TNF) receptorassociated factor 4 (TRAF4), TRAF6, TGF- $\beta$ activated kinase 1 (TAK1 or MAP3K), p38 mitogen-activated protein kinase 1 (p38 MAPK), RHO, phosphoinositide 3-kinase (PI3K), AKT (also known as protein kinase B), extracellular signalregulated kinase (ERK), JUN N-terminal kinase (JNK) or nuclear factor $\kappa \mathrm{B}(\mathrm{NF}-\mathrm{\kappa} \mathrm{B})$. The mode of action and outcome varies according to the activation of the individual pathway. 
Among the other non-canonical pathways in TGF- $\beta$ signaling, the RAS-ERK pathway has been shown to involved in many different points of carcinogenesis. Primarily ERK functions as proto-oncogene as it promotes cell proliferation, migration, and tumor metastasis but at some points, it antagonizes the tumorigenesis. In breast cancer, ERK inhibits cell apoptosis and in pancreatic cancer, ERK upregulates p 21 and thereby facilitates TGF- $\beta$ mediated cell cycle arrest ${ }^{30,31}$. Sustained existence of ERK could facilitate cell progression through entering S-phase of cell cycle ${ }^{32}$. ERK contributes to the TGF- $\beta$ signaling pathway in different stages; antagonizing the SMAD signaling through phosphorylation of SMAD2 and SMAD3 at SMAD linker region and facilitating the SMAD signaling through phosphorylation of other SMAD3 residues ${ }^{33-36}$. Principe et at. showed ERK upregulation in pancreatic cancer cells and their study also found ERK-induced cell cycle arrest through TGF- $\beta /$ SMAD4 mediated p21 upregulation in benign pancreatic cell line ${ }^{34}$. The author and his group demonstrated the role by showing the reduced level of TGF- $\beta$ mediated nuclear translocation of SMAD4 and p21 while pharmacologically pretreated with ERK antagonist, in benign and well-differentiated neoplastic pancreatic ductal epithelial cell ${ }^{37}$. In advanced pancreatic cancer cell phosphorylated ERK has no regulatory effect on TGF- $\beta$ induced upregulation of p21 and nuclear translocation but it plays a crucial role in TGF- $\beta$ mediated EMT in all pancreatic cancer cell lines ${ }^{34}$. Like TGF- $\beta$, ERK contributes to the carcinogenesis in various ways and some of its contradictory functions brought it as a topic of further investigation.

In advanced pancreatic cancer overexpression of TGF- $\beta$ and loss of SMAD4 function is one of the explanations of directing the SMAD-independent pathway. A study has shown that TGF- $\beta$ could still act as tumorigenesis while SMAD4 RNA was downregulated pancreatic cancer cell lines $^{38}$. The switching of TGF- $\beta$ to SMAD independent pathway could also be related to the SMAD4 requirement. Further studies are required for a better understanding of this cross over function of TGF- $\beta$ in carcinogenesis.

\section{TGF-B/SMAD4, Tumor Suppression to Progression}

The cell cycle inhibition or growth inhibitory effect of TGF- $\beta /$ SMAD4 signaling pathway defines its tumor suppressive role whereas in carcinogenesis overexpression of TGF- $\beta$ and loss of TGF- $\beta /$ SMAD4 mediated tumor suppressive action leads to tumor progression ${ }^{18}$. The other crucial roles of TGF- $\beta$ in cancer progression are; immune suppression, angiogenesis, and epithelial-mesenchymal transition (EMT) ${ }^{39,40}$.

In early neoplastic and benign pancreatic epithelial cells, cell cycle arrest by TGF- $\beta /$ SMAD4 is the main tumor suppressive function, mediated by upregulation of targets like; $21^{41,42}$. In the cell cycle transition from G1 to S phase is inhibited by cyclin-dependent kinase $\mathrm{p} 21^{43}$. Upregulation of p 21 is mediated by TGF- $\beta$ /SMAD4 pathway while nonSMAD also regulate p21 as seen in colon cancer $^{44,45}$. In a pancreatic cancer patient, high levels of p21 is an indicator of good prognosis as it inhibits cell proliferation as well as acinar to ductal metaplasia ${ }^{46}$. Loss of SMAD4 in PDAC opposes the cell cycle regulation and favor proliferation. The similar result is seen in TGF- $\beta$ mediated apoptosis effect in the tumor microenvironment. TGF- $\beta$ regulates expression of different genes through SMAD4 like; GADD45 $\beta \mathrm{r}$ signaling factor domain, the bcl-2 Bim homologous factor, the death-associated protein kinase, and TGF$\beta$-inducible early response gene 1 (TIEGI) ${ }^{47}$. A genetic mutation in TGF- $\beta$ signaling components or changes in signaling pathways often downregulates apoptosis and cell cycle arrest and leads to cancer progression.

In carcinogenesis, EMT is an important transition that leads to the progression and metastasis of cancer cells ${ }^{39}$. In benign and cancer cells EMT is mediated by a complex of cellular and molecular changes in transcriptional and translational levels ${ }^{48,49}$. Depending on the cell type and tissue microenvironment these changes downregulate expression of epithelial junctional proteins and markers and upregulate mesenchymal adhesion and marker protein $s^{50}$. The ultimate transcription factor families that regulate the transition are Snail/Slug, Zeb1/2, and twist family $^{51}$. In the TGF- $\beta /$ SMAD4-dependent pathway, the SMAD3/SMAD4 complex induces transcription of Snail protein and decreases expression of epithelial junction protein E-cadherin and occludin ${ }^{52,53}$. TGF- $\beta$ also increases the expression of ZEB transcription factors through SMAD4dependent pathway and decrease expression of miR-200 family, T $\beta R 1$ and SMAD2, which reflexly increase ZEB1 and ZEB2 mRNA and protein levels and thus further bolstering the EMT response ${ }^{53,54}$. In the late stage of tumorigenesis, TGF- $\beta$ promotes tumor growth by a combined effect of SMAD4-dependent and SMAD-independent effects on $\mathrm{EMT}^{5,55}$. The critical role of TGF- $\beta$ in cancer dissemination is complex and extensively studied to emphasize that TGFdrives cancer progression not only by cell growth but also and more importantly by $\mathrm{EMT}^{40}$.

In addition to its effect on tumor growth, TGF- $\beta$ modulates tumor development and progression by changing the tumor microenvironment ${ }^{56}$. TGF- $\beta$ suppresses immune and inflammatory processes through the inhibition of CD8+ cytotoxic T cells, macrophages, dendritic cells, and $\mathrm{NK}$ cells ${ }^{56}$. It has recently been found that sustained activation of pro-inflammatory transcription factor NF- $\mathrm{KB}$ in PDAC is closely involved in driving tumor progression. Intact SMAD4, S100A8, S100A9, and S100A8/A9 share an overall inhibitory effect on NF- $\kappa B$, while these molecules do not affect NF- $\mathrm{KB}$ in the presence of SMAD4 homozygous 
deletion. Thus, SMAD4 plays a key role in suppressing the inflammatory signaling cascades important in PDAC ${ }^{57}$.

PDAC has a strong effect on the deposition of extracellular matrix (ECM), eliciting a strong desmoplastic reaction. In addition to ECM, endothelial cells, immune cells, and fibroblasts are recruited to the tumor microenvironment and thus contribute to both tumor growth and invasion ${ }^{58,59}$. TGF- $\beta$ expression enhances the release of multiple ECM molecules including fibronectin, collagen fibulins, and elastin ${ }^{60,61}$. Overexpression of TGF- $\beta$ is a critical mediator of fibrosis in many tumors ${ }^{62-64}$. TGF- $\beta$ also inhibits the degradation of newly synthesized ECM by inhibiting the synthesis of matrix metalloproteinase (MMP) and by inhibiting the expression of genes responsible to produce MMP ${ }^{65}$. T $\beta R I I$ is also known to be overexpressed in PDAC and correlate with advanced tumor stage, decreased patient survival, and increased expression of genes known to promote angiogenesis and invasion (e.g., plasminogen activator 1 and matrixmetalloproteinase-9) ${ }^{66-68}$. Additionally, high levels of SMAD2 have been documented in $\mathrm{PDAC}^{68}$, leading to a more potent response to TGF- $\beta$ signals.

The correlation of TGF- $\beta$ overexpression and cancer progression is established in several malignancies ${ }^{69,70}$. An elevated level of TGF- $\beta$ is found in both tissue and plasma of PDAC patients and is correlated with the presence of metastases in CRC, PDAC, as well as prostate and breast cancers $^{69}$. Additionally, cancer cells, in general, have been shown to secrete higher amounts of TGF- $\beta$ than their normal cell counterparts. Conversely, reduced levels of circulating TGF- $\beta$ isoforms in patient serum was found to be associated with prolonged survival ${ }^{71}$.

SMAD4 gene inactivation is associated with poor prognosis in clinically diagnosed PDAC cases, due to an increased incidence of metastatic disease. Studies by Blackford et al. have shown that SMAD4 mutation was more prevalent in unresectable PDAC cases with a higher metastatic burden, whereas SMAD4 wildtype cases were more amenable to chemotherapy and surgical resection ${ }^{70}$. Furthermore, clinical studies have proved that SMAD4 protein expression, acts as an important prognostic marker. In a series of over 200 PDAC cases, median survival was shown to be 19.2-20.5 months for intact SMAD4 expression compared to 13.7-14.7 months for loss of SMAD4 expression, respectively ${ }^{67}$. Several meta-analyses have also proven the association of SMAD4 loss with poor prognosis $^{15,67,70}$.

SMAD4 inactivating mutations occur in approximately $20 \%$ of all pancreatic cancer. They are typically within the MH1 (DNA binding) or MH2 (transcriptional activation) domains of the protein. Additional mutations include deletion of the entire chromosome with a point, frameshift, nonsense, and missense mutations reported. Functional studies have shown missense mutations within the MH2 domain result in the loss of protein stability and disruption of the dimerization ability of the SMADS ${ }^{72}$. Furthermore, a study by $\mathrm{Xu}$ etal. (2000) found that although SMAD4 proteins with MH1 domain point mutations are translated at similar rates as wild-type proteins, they have a shorter half-life and are more rapidly degraded by a ubiquitinmediated pathway ${ }^{72}$.

\section{SMAD4 as a Prognostic Marker}

The diagnosis of pancreatic adenocarcinoma frequently occurs at an advanced stage, leading to inoperability and a mediansurvival rate of 4-8 months following diagnosis. When the disease is limited to the pancreas, surgical resection can improve the 5-year survival rate to $15-20 \%$. The surgical and pathological prognostic factors for PDAC are tumor location (localized in the pancreas), margin status, vascular invasion, tumor size, and TNM staging. Recent studies have proven that tumor expression of TGF- $\beta$ /SMAD4 plays a pivotal role in cancer prognosis and survival of the patient. More than $50 \%$ of PDAC show SMAD4 alteration with concomitant mutation of INK4A/ARF tumor suppressor gene and a Kras oncogene ${ }^{5}$. A SMAD4 related survival study of 25 PDAC patients (8 patients with gene alteration) show SMAD4 gene mutated cases survival period was shorter than SMAD4 nonmutated cases (median survival 5 and 10 months, respectively, $\mathrm{P}=0.001)^{73}$. In a different study of surgically resected pancreatic adenocarcinoma patients, survival was shown significantly longer (unadjusted median survival, 19.2 months) with tumors expressed SMAD4 protein as compared with SMAD4 protein expression (14.7 months, $\mathrm{P}_{\text {_ }}$ 0.03). This SMAD4 survival benefit was not related to other prognostic factors including tumor size, margin status, lymph node status, pathological stage, blood loss, and use of adjuvant chemoradiotherapy ${ }^{10}$. The median survival differences of about 5 months explain the clinical significance of SMAD4 expression PDAC. A meta-analysis of 4247 patients in 20 published articles concluded that the immunohistochemical loss of SMAD4 predicted poor overall survival in both Asian and Caucasian patients with pancreatic cancer, but did not correlate with tumor size, differentiation, or lymph node metastasis ${ }^{68}$. In another metaanalysis of 1762 patients from 14 studies found that loss of SMAD4 correlated significantly with poor overall survival. The multivariate analysis showed that the loss of SMAD4 predicted poor prognosis in patients with less advanced disease (likely Stage I to Stage II pancreatic cancer) ${ }^{15}$.

A recent development in gene sequencing technology allows us to know the detail of cancer genome and to identify genetic markers for prognosis of pancreatic cancer. Blackford et.al studied a series of 24 adenocarcinoma patients by doing protein-coding gene sequencing. The result showed a mutation of 39 genes in more than one of these cases. In their next study, they showed somatic 
mutation correlated with the survival of the patients. They examined all the members of the TGF- $\beta$ pathway (SMAD3, SMAD4, TGF $\beta$ R1, and TGF $\beta$ R2) individually and together. The survival analysis of mutated and non-mutated SMAD4 gene showed a significant difference. Cases with the intact SMAD4 gene had a median survival rate longer than SMAD4 mutated gene (14.2 [95\% C.I., 12.5 - 20.5 and 11.5 [95\% C.I., 8.5 - 16.0] month respectively) ${ }^{70}$. The recent extensive molecular study is going on to definitively define the subgroups of pancreatic cancer who could be benefited by targeted therapy.

\section{Current Targeted Therapy for the TGF- $\beta$ /SMAD Pathway}

Role of TGF- $\beta$ signaling as a potent tumor suppressor in most of the solid tumors focus on developing the therapeutic target. In advanced cancers, there is a selective loss of TGF- $\beta$ induce growth inhibitory function and induction of other activities that lead to growth, invasion, and metastasis of cancer cells ${ }^{56,69}$. Current studies differentiate the tumor suppressive and promoting the role of TGF- $\beta$ at the cellular level to potentiate the anti-tumor effect and at the same time suppress the pro-oncogenic effect. TGF- $\beta /$ SMAD dependent signaling pathway is thought to be the tumor suppressive whereas the other TGF- $\beta$ /SMAD independent pathway is considered as pro-oncogenic. The dual functions of TGF- $\beta$ bring a challenge to identify the selective pathway of the tumor for the appropriate therapeutic intervention. There are several proposed therapeutic approaches to target the TGF- $\beta$ pathway. According to Massage et al, three best approaches to inhibit TGF- $\beta$ signaling pathways are 1) translational level inhibition, 2) ligand-receptor level inhibition and 3) inhibition of receptor-mediated signaling ${ }^{69}$. The aim of each of these targeted therapies is to inhibit tumor-promoting function and maintain the tumor suppressive function of TGF- $\beta$. One of the other conventional approaches is to target TGF- $\beta$ induced epithelial-mesenchymal transition (EMT). Advancement of large molecule inhibitors and small molecule inhibitors are designed to inhibit the generation of TGF- $\beta$ and downstream signaling pathway.

AP-12009, one of the antisense oligonucleotides (ASO) act directly against the mRNA of TGF- $\beta 2$. An ongoing study has shown a survival benefit of pancreatic adenocarcinoma and melanoma using AP-12009, supporting the promising role of AP-12009 on aggressive tumors overexpress TGF- $\beta 2^{74}$. The other ASO under study and preclinical training are AP-11014 and AP-15012 ${ }^{28}$. Monoclonal antibodies are the choice of targeted therapy for many cancers. In the TGF- $\beta$ signaling pathway, these antibodies target the ligand-receptor binding and prevent subsequent ligand signaling ${ }^{28}$. Some of these monoclonal antibodies are in under advance the clinical investigation, including Lerdelimumab (CAT-152, Trabio TM), metelimumab (CAT-
192) antibody against TGF- $\beta 2$, TGF- $\beta 1$. In tumor immune microenvironment, TGF- $\beta$ exerts its immunosuppressive function through antagonizing Interleukin (IL)-15 mediated natural killer (NK) cell proliferation. The suppression of the cytotoxic function of NK and T cells, through SMAD dependent pathway, is another key role in tumor immune evasion ${ }^{75,76}$. Inhibition of TGF- $\beta$ mediated signaling has shown a reversal of immune evasion function by the restoration of immune activity against the tumor cells ${ }^{77}$. Targeting TGF- $\beta$ mediated immunosuppression in the tumor microenvironment is a new therapeutic target but the effects of the TGF- $\beta$ inhibitor on human immune system needs to be further evaluated.

The dual role of TGF- $\beta$ in oncogenesis makes it a potential target for cancer therapy. Extensive studies have been done to evaluate its nature of interactions with TGF$\beta$-receptors and subsequent signaling pathway (SMAD dependent and SMAD independent). Preclinical and clinical studies are going on targeting at different levels of interactions are showing promising results.

\section{Conclusion}

PDAC is a devastating malignancy highlighted by the early metastatic spread and advanced stage at diagnosis. Most of the cases are not amenable to surgical resection. Several studies have shown a strong association of TGF- $\beta$ with cancer prognosis, metastasis, and survival. Additionally, the role of SMAD-dependent signal transduction pathway is important to explain the association of TGF- $\beta$ with PDAC carcinogenesis. TGF- $\beta$ targeted therapy is now established for several human cancers and several preclinical and clinical data have shown that TGF- $\beta$ blockade could be effective in the treatment of PDAC. Checkpoint inhibitors introduce a new therapeutic guideline in treating cancer. Monotherapy with checkpoint inhibitors could not sufficiently treat an advanced pancreatic cancer patient. There is increasing evidence of treating several cancers with targeting TGF-B signaling suggesting the combination of check-point inhibitors with TGF-B signaling inhibition could increase the survival. Thusly, the precision targeting of the TGF- $\beta$ /SMAD4 signaling pathway could be critical in the treatment of PDAC.

\section{Acknowledgments}

The cost for publication is supported in part by the Department of Pathology, NYU School of Medicine/ Langone Medical center. The authors thank the personnel of Department of Experimental Pathology, NYU Langone Medical Center for their assistance.

\section{Author Contributions}

S.A. and R.U. formulated the original hypothesis, designed the study and wrote the manuscript. C.S and M.Z.D wrote the manuscript. 


\section{References}

1. Li D, Xie K, Wolff R, Abbruzzese JL. Lancet. 2004; 363 (9414): 10491057.

2. Gong Z, Holly EA, Bracci PM. Survival in population-based pancreatic cancer patients: San Francisco Bay area, 1995-1999. Am J Epidemiol. 2011; 174: 1373-1381.

3. Sultana A, Cox T, Ghaneh P, et al. Adjuvant therapy for pancreatic cancer. Recent Results Cancer Res. 2012; 196: 65-88.

4. Ali S, Cohen C, Little JV, et al. The utility of SMAD4 as a diagnostic immunohistochemical marker for pancreatic adenocarcinoma, and its expression in other solid tumors. Diagn Cytopathol. 2007; 35 (10): 644-648.

5. Hahn SA, Schutte M, Hoque AT, et al. DPC4, a candidate tumor suppressor gene at human chromosome 18q21.1. Science. 1996; 271 (5247): 350-353.

6. Hahn SA, Hoque AT, Moskaluk CA, et al. Homozygous deletion map at 18q21.1 in pancreatic cancer. Cancer Res. 1996; 56 (3): 490-494.

7. Schutte M, Hruban RH, Hedrick L, et al. DPC4 gene in various tumor types. Cancer Res. 1996; 56 (11): 2527-2530.

8. Hruban RH, Wilentz RE, Kern SE. Genetic Progression in the Pancreatic Ducts. Am J Pathol. 2000; 156 (6): 1821-1825.

9. Furukawa T, Sunamura M, Horii A. Molecular mechanisms of pancreatic carcinogenesis. Cancer Sci. 2006; 97: 1-7.

10. Xia X, Wu W, Huang C, et al. SMAD4 and its role in pancreatic cancer Tumour Biol. 2015; 36: 111-119.

11. Pérez-Mancera PA, Guerra C, Barbacid M, et al. What we have learned about pancreatic cancer from mouse models. Gastroenterology. 2012; 142: 1079-1092.

12. Bardeesy $\mathrm{N}$, Cheng $\mathrm{KH}$, Berger $\mathrm{JH}$, et al. Smad4 is dispensable for normal pancreas development yet critical in progression and tumor biology of pancreas cancer. Genes Dev. 2006; 20: 3130-3146.

13. Ijichi H, Chytil A, Gorska AE, et al. Aggressive pancreatic ductal adenocarcinoma in mice caused by pancreas-specific blockade of transforming growth factor-beta signaling in cooperation with active Kras expression. Genes Dev. 2006; 20: 3147-3160.

14. Izeradjene $\mathrm{K}$, Combs $\mathrm{C}$, Best $\mathrm{M}$, et al. Kras ${ }^{\mathrm{G} 12 \mathrm{D}}$ and Smad4/Dpc4 haploinsufficiency cooperate to induce mucinous cystic neoplasms and invasive adenocarcinoma of the pancreas. Cancer Cell. 2007; 11: 229-243.

15. Xing S, Yang H, Liu J, et al. Prognostic Value of SMAD4 in Pancreatic Cancer: A Meta-Analysis. Transl Oncol. 2016; 1: 1-7.

16. Moore PS, Sipos B, Orlandini S, et al. Genetic profile of 22 pancreatic carcinoma cell lines. Analysis of K-ras, p53, p16, and DPC4/Smad4. Virchows Arch. 2001; 439: 798-802.

17. Tirado-Rodriguze B, Ortega E, Segura-Medina P, et al. TGF-?: An Important mediator of allergic disease and a molecule with dual activity in cancer development. J Immunol Res. 2014; 318481: 1-15.

18. Derynck R, Zhang YE. Smad-dependent and Smad-independent pathways in TGF-beta family signalling. Nature. 2003; 425: 577-584.

19. Lack J, O'Leary JM, Knott V, et al. Solution structure of the third TB domain from LTBP1 provides insight into assembly of the large latent complex that sequesters latent TGF-beta. J Mol Biol. 2003; 334: 281291.

20. Shi Y, Massagué J. Mechanisms of TGF- $\beta$ signaling from cell membrane to the nucleus. Cell. 2003; 113: 685-700.

21. Zhang Y, Musci T, Derynck R. The tumor suppressor Smad4/DPC 4 as a central mediator of Smad function. Curr Biol. 1997; 7: 270-276.

22. Chen Y, Lebrun JJ, Vale W. Regulation of transforming growth factor $\beta$ - and activin-induced transcription by mammalian Mad proteins. Proc Natl Acad Sci USA. 1996; 93: 12992-12997.

23. Zhang Y, Feng XH, Derynck R. Smad3 and Smad4 cooperate with c-Jun/c-Fos to mediate TGF-beta-induced transcription. Nature. 1998; 394: 909-913.

24. Biswas S, Criswell TL, Wang SE, et al. Inhibition of transforming growth factor-beta signaling in human cancer: targeting a tumor suppressor network as a therapeutic strategy. Clin Cancer Res. 2006; 12: 4142-4146.

25. Jakowlew SB. Transforming growth factor-beta in cancer and metastasis. Cancer Metastasis Rev. 2006; 25: 435-457.

26. Furukawa T, Sunamura M, Horii A. Molecular mechanisms of pancreatic carcinogenesis. Cancer Sci. 2006; 97: 1-7.

27. Duda DG, Sunamura M, Lefter LP, et al. Restoration of SMAD4 by gene therapy reverses the invasive phenotype in pancreatic adenocarcinoma cells. Oncogene. 2003; 22: 6857-6864.

28. Gomez-Puerto MC, Iyengar PV,Vinuesa AGD, et al. Bone morphogenetic protein receptor signal transduction in human disease. J Pathol. 2019; 247: 9-20.

29. Zhang YE. Non-Smad signaling pathways of the TGF- $\beta$ family. Cold Spring Harb Perspect Biol. 2017; 9: a022129.

30. Tang D, Wu D, Hirao A, et al. ERK activation mediates cell cycle arrest and apoptosis after DNA damage independently of p53. J Biol Chem. 2002; 277: 12710-12717.

31. Torii S, Yamamoto T, Tsuchiya Y, et al. ERK MAP kinase in G cell cycle progression and cancer. Cancer Sc. 2006; 97: 697-702.

32. Pouyssegur J, Volmat V, Lenormand P. Fidelity and spatio-temporal control in MAP kinase (ERKs) signalling. Biochem Pharmaco. 2002; 64: 755-763.

33. Lee MK, Pardoux C, Hall MC, et al. TGF-beta activates Erk MAP kinase signalling through direct phosphorylation of ShcA. EMBO J. 2007; 26: 3957-3967.

34. Principe DR, Diaz AM, Torres $C$, et al. TGF $\beta$ engages MEK/ERK to differentially regulate benign and malignant pancreas cell function. Oncogene. 2017; 36: 4336-4348.

35. Hough C, Radu M, Doré JJ. TGF-beta induced Erk phosphorylation of smad linker region regulates smad signaling. PLoS ONE. 2012; 7 : e42513.

36. Ellenrieder V, Hendler SF, Boeck W, et al. Transforming growth factor beta1 treatment leads to an epithelial-mesenchymal transdifferentiation of pancreatic cancer cells requiring extracellular signal-regulated kinase 2 activation. Cancer Res. 2001; 61: 42224228.

37. Principe DR, DeCant B, Staudacher J, et al. Loss of TGFbeta signaling promotes colon cancer progression and tumor-associated inflammation. Oncotarget. 2017; 8: 3826-3839.

38. Wrighton KH, Lin X, Feng XH. Phospho-control of TGF-beta superfamily signaling. Cell Res. 2009; 19: 8-20.

39. Leung L, Radulovich N, Zhu CQ et al. Loss of canonical Smad4 signaling promotes KRAS driven malignant transformation of human pancreatic duct epithelial cells and metastasis. PLoS ONE. 2013; 8:e84366

40. Krantz SB, Shields MA, Dangi-Garimella S, et al. Contribution of epithelial-to-mesenchymal transition and cancer stem cells to pancreatic cancer progression. J Surg Res. 2012; 173: 105-112.

41. Principe DR, Doll JA, Bauer J, et al. TGF-beta: duality of function between tumor prevention and carcinogenesis. J Natl Cancer Inst. 2014; 106: djt369.

42. Nicolas FJ, Hill CS. Attenuation of the TGF-beta-Smad signaling 
pathway in pancreatic tumor cells confers resistance to TGF-betainduced growth arrest. Oncogene. 2003; 22: 3698-3711.

43. Gartel AL, Radhakrishnan SK. Lost in transcription: p21 repression, mechanisms, and consequences. Cancer Res. 2005; 65: 3980-3985.

44. Yasutome M, Gunn J, Korc M. Restoration of Smad4 in BxPC3 pancreatic cancer cells attenuates proliferation without altering angiogenesis. Clin Exp Metastasis. 2005; 22: 461-473.

45. Ijichi H, Otsuka M, Tateishi K, et al. Smad4-independent regulation of p21/WAF1 by transforming growth factor-beta. Oncogene. 2004; 23 1043-1051.

46. Grabliauskaite K, Hehl AB, Seleznik GM, et al. p21(WAF1) (/Cip1) limits senescence and acinar-to-ductal metaplasia formation during pancreatitis. J Pathol. 2015; 235: 502-514.

47. Yin JJ, Selander K, Chirgwin JM, et al. TGF- $\beta$ signaling blockade inhibits PTHrP secretion by breast cancer cells and bone metastases development. J Clin Invest. 1999; 103: 197-206.

48. Thiery JP, Acloque H, Huang RY, et al. Epithelial-mesenchymal transitions in development and disease. Cell. 2009; 139:871-890.

49. Yang J, Weinberg RA. Epithelial-mesenchymal transition: at the crossroads of development and tumor metastasis. Dev Cell. 2008; 14: 818-829.

50. Thiery JP, Sleeman JP. Complex networks orchestrate epithelialmesenchymal transitions. Nat Rev Mol Cell Biol. 2006; 7: 131-142.

51. Peinado H, Olmeda D, Cano A. Snail, Zeb and bHLH factors in tumour progression: an alliance against the epithelial phenotype? Nat Rev Cancer. 2007; 7: 415-428.

52. Meulmeester E, Ten Dijke P. The dynamic roles of TGF- $\beta$ in cancer. J Pathol. 2011; 223: 205-218.

53. Vincent T, Neve EP, Johnson JR, et al. A SNAIL1-SMAD3/4 transcriptional repressor complex promotes TGF- $\beta$ mediated epithelial-mesenchymal transition. Nat Cell Biol. 2009; 11: 943-950.

54. Bracken CP, Gregory PA, Kolesnikoff N, et al. A double-negative feedback loop between ZEB1-SIP1 and the microRNA-200 family regulates epithelial-mesenchymal transition. Cancer Res. 2008; 68: 7846-7854.

55. Derynck R, Akhurst RJ. Differentiation plasticity regulated by TGF- $\beta$ family proteins in development and disease. Nat Cell Biol. 2007; 9: 1000-1004.

56. Sánchez-Elsner T, Botella LM, Velasco B, et al. Synergistic cooperation between hypoxia and transforming growth factor-beta pathways on human vascular endothelial growth factor gene expression. J Biol Chem. 2001; 276: 38527-38535.

57. Gurumurthy S, Bardeesy N. Uncapping NF- $\mathrm{KB}$ activity in pancreatic cancer. EMBO J. 2011; 30: 1-2.

58. Eliceiri BP. Integrin and growth factor receptor crosstalk. Circ Res. 2001; 89: 1104-1110

59. Weis SM, Cheresh DA. Tumor angiogenesis: Molecular pathways and therapeutic targets. Nat Med. 2011; 17: 1359-1370.

60. Mao Y, Keller ET, Garfield DH, et al. Stromal cells in tumor microenvironment and breast cancer. Cancer Metastasis Rev. 2013; 32: 303-315.
61. Kuang PP, Joyce-Brady M, Zhang XH, et al. Fibulin-5 gene expression in human lung fibroblasts is regulated by TGF- $\beta$ and phosphatidylinositol 3-kinase activity. Am J Physiol. Cell Physiol. 2006; 291: C1412-C1421.

62. Krzemień S, Knapczyk P. Current review on the role of transforming growth factor beta (TGF-beta) in some pathological disorders. Wiad Lek. 2005; 58: 536-539.

63. Ignotz RA, Massagué J. Transforming growth factor-beta stimulates the expression of fibronectin and collagen and their incorporation into the extracellular matrix. J Biol Chem. 1986; 261: 4337-4345.

64. Kajdaniuk D, Marek B, Borgiel-Marek H, et al. Vascular endothelial growth factor (VEGF)-Part 1: In physiology and pathophysiology. Endokrynol Polska. 2011; 62: 444-455.

65. Kajdaniuk D, Marek B, Foltyn W, et al. Vascular endothelial growth factor (VEGF)-Part 2: In endocrinology and oncology. Endokrynol Polska. 2011; 62: 456-464

66. Zhao J, Liang Y, Yin Q, et al. Clinical and prognostic significance of serum transforming growth factor-beta1 levels in patients with pancreatic ductal adenocarcinoma. Braz J Med. Biol Res. 2016; 49 (8): e5485.

67. Tascilar M, Skinner HG, Rosty C, et al. The Smad4 protein and prognosis of pancreatic ductal adenocarcinoma. Clin Cancer Res. 2001; $12:$ 4115-4121.

68. Du Y, Zhou X, Huang Z, et al. Meta-Analysis of the Prognostic Value of Smad4 Immunohistochemistry in Various Cancers. PLoS ONE. 2014; 10: e110182.

69. Massagué J. TGFß in Cancer. Cell. 2008; 134: 215-230.

70. Blackford A, Serrano OK, Wolfgang CL, et al. SMAD4 gene mutations are associated with poor prognosis in pancreatic cancer. Clin Cancer Res. 2009; 15: 4674-4679.

71. Maitra A, Molberg K, Albores-Saavedra J, et al. Loss of Dpc4 expression in colonic adenocarcinomas correlates with the presence of metastatic disease. Am J Pathol. 2000; 157: 1105-1111.

72. Xu J, Attisano L. Mutations in the tumor suppressors Smad2 and Smad4 inactivate transforming growth factor $\beta$ signaling by targeting Smads to the ubiquitin-proteasome pathway. Proc Natl Acad Sci U S A. 2000 ; 97 : $4820-4825$.

73. Bilici A. Prognostic factors related with survival in patients with pancreatic adenocarcinoma. World J Gastroenterol. 2014; 20: 1080210812.

74. Nemunaitis J, Jahan $\mathrm{T}$, Ross $\mathrm{H}$, et al. Phase $1 / 2$ trial of autologous tumor mixed with an allogeneic GVAX vaccine in advanced-stage nonsmall-cell lung cancer. Cancer Gene Ther. 2006; 13: 555-562.

75. Trotta R, Col JD, Yu J, et al. TGF-beta utilizes SMAD3 to inhibit CD16mediated IFN-gamma production and antibody-dependent cellular cytotoxicity in human NK cells. J Immunol. 2008; 181: 3784-3792.

76. Thomas DA, Massague J. TGF-beta directly targets cytotoxic T cell functions during tumor evasion of immune surveillance. Cancer Cell. $2005 ; 8: 369-380$.

77. Wilson EB, El-Jawhari JJ, Neilson AL, et al. Human tumour immune evasion via TGF-beta blocks NK cell activation but not survival allowing therapeutic restoration of anti-tumour activity. PLoS One. 2011; 6: e22842. 